\title{
$\gamma$-Glutamyl transpeptidase catalyses the extracellular detoxification of cisplatin in a human cell line derived from the proximal convoluted tubule of the kidney
}

\author{
A. Paolicchi ${ }^{\mathrm{a}}$, M. Sotiropuolou ${ }^{\mathrm{b}}$, P. Perego ${ }^{\mathrm{c}}$, S. Daubeuf ${ }^{\mathrm{d}}$, A. Visvikis ${ }^{\mathrm{e}}$, E. Lorenzini $^{\mathrm{a}}$, \\ M. Franzini ${ }^{\mathrm{a}}$, N. Romiti ${ }^{\mathrm{a}}$, E. Chieli ${ }^{\mathrm{a}}$, R. Leone ${ }^{\mathrm{c}}$, P. Apostolif ${ }^{\mathrm{f}}$, D. Colangelo ${ }^{\mathrm{g}}$, F. Zunino ${ }^{\mathrm{c}}$, \\ A. Pompella ${ }^{\mathrm{a}, *}$ \\ ${ }^{a}$ Dipartimento di Patologia Sperimentale, sez. Patologia Generale e Clinica, Università di Pisa, via Roma 55, Pisa, Italy \\ ${ }^{\mathrm{b}}$ Department of Pharmacology, University of Ioannina, Medical School, 451-10 Ioannina, Greece \\ ${ }^{c}$ Istituto per lo Studio e la Cura dei Tumori, via Venezian, 1, Milan, Italy \\ ${ }^{\mathrm{d}}$ Thiols and Fonctions Cellulaires, Facultè de Farmacie, University of Nancy, rue Lionnois, 30, BP 403, Nancy, France \\ e Istituto di Farmacologia, Università di Verona, Policlinico Borgo Roma, 37124 Verona, Italy \\ ${ }_{\mathrm{f}}^{\mathrm{f}}$ Istituto di Medicina del Lavoro, Università di Brescia, 25123 Brescia, Italy \\ ${ }^{\mathrm{g}}$ Dipartimento di Scienze Mediche, Università del Piemonte Orientale, via Solaroli 17, 28100 Novara, Italy
}

Received 12 July 2002; received in revised form 7 October 2002; accepted 11 November 2002

\begin{abstract}
Nephrotoxicity is a side-effect and the main factor limiting the clinical use of cisplatin. In vivo, the administration of the cysteinecontaining tripeptide glutathione (GSH) has been found to reduce nephrotoxicity, but the biochemical mechanism of this protective action is not fully understood. The present study was designed to gain insights into the mechanism by which GSH prevents cisplatin nephrotoxicity. We also wanted to verify the hypothesis of whether the protective action of GSH is mediated by products of the extracellular breakdown of GSH catalysed by $\gamma$-glutamyl transpeptidase (GGT), an enzyme that is highly expressed in kidney tubular cells. The study was performed in HK-2 cells, derived from the immortalisation of human kidney proximal tubule cells. We investigated the influence of modulators of GGT activity and/or thiols on the antiproliferative activity of cisplatin and on the intracellular GSH content. We determined the antiproliferative activity of cisplatin, platinum cellular accumulation and DNA platination following precomplexing of the drug with thiols. The antiproliferative effect of cisplatin was minimally affected by the addition of GSH. However, when the antiproliferative assay was performed in the presence of glycyl-glycine (GlyGly), to serve as a transpeptidation acceptor and thus to stimulate GGT-mediated GSH catabolism, cisplatin-induced growth inhibition was largely prevented. This effect was not mediated through an increase of intracellular GSH levels, which were not affected by the GlyGly supplementation. The thiol dipeptide cysteinyl-glycine, i.e. the GSH catabolite generated by GGT activity, showed a higher reactivity against cisplatin in vitro than GSH, as was shown by the more rapid oxidation of its - $\mathrm{SH}$ groups. The cisplatin/GSH or cisplatin/cysteinyl-glycine adducts did not display an antiproliferative effect. However, $2 \mathrm{~h}$ precomplexing with GSH in the presence of GGT, or directly with the GSH catabolite cysteinyl-glycine, decreased the antiproliferative effect of cisplatin and drug-induced DNA platination to a greater extent than precomplexing with GSH alone. The results of the present study show that, in HK-2 cells, extracellular GSH decreases the antiproliferative effects of cisplatin only upon its hydrolysis by GGT, thereby supporting the hypothesis that the extracellular metabolism of GSH by GGT plays a role in modulating cisplatin nephrotoxicity. A primary role in the protection of HK-2 cells appears to be played by cysteinyl-glycine, the proximal product of the GGT-mediated hydrolysis of GSH, which shows a high reactivity against CDDP resulting in the rapid inactivation of the drug.

(C) 2003 Elsevier Science Ltd. All rights reserved.
\end{abstract}

Keywords: Cisplatin; Kidney tubular cells; $\gamma$-Glutamyl transpeptidase; Toxicity

* Corresponding author. Tel. + 39-050-554851; fax + 39-050-554929.

E-mail address: apompella@biomed.unipi.it (A. Pompella). 


\section{Introduction}

Cisplatin (cis-diammine-dichloro-platinum, CDDP) is a widely used anticancer drug, exhibiting efficacy against a variety of solid human cancers, including carcinoma of ovary, lung, head and neck, bladder and testis [1]. CDDP enters cells and reacts with nucleophilic sites in cellular macromolecules [2]. The presence of CDDP adducts in DNA can trigger cell cycle arrest and apoptosis [3]. The sensitivity of individual cell types to the effects of CDDP is thought to depend on the specific balance between drug uptake and accumulation, binding to DNA and the capability of repairing damaged portions of DNA [3]. In addition to the appearance of intrinsic resistance of cells to the drug, the efficacy of CDDP is limited by its side-effects, among which nephrotoxicity is the most common [4]. Likely due to reabsorption of urine components, platinum is rapidly accumulated in the kidney after CDDP administration, reaching higher concentrations than in other organs, and is retained there for several days. This may be the main cause of the organ-specific toxicity, which mainly affects the proximal convoluted tubule [5].

As the precise mechanism of CDDP uptake in the kidney has not yet been identified, a corresponding therapy targeted at the mechanism of this CDDPinduced nephrotoxicity is not available. Several amino acids have been tested in vivo as possible inhibitors of CDDP nephrotoxicity, and the sulphur-containing amino acids L-cysteine [6], L-methionine [7] and $N$-acetylcysteine [8] have shown the highest activity. Studies in vitro on immortalised mouse kidney tubule cells showed that extracellular cysteine (followed by methionine) possesses the highest protective effect [9]. The mechanism of action of extracellular cysteine is apparently multiphasic, involving the inhibition of CDDP uptake, as well as a direct reaction with CDDP to form adducts; the cysteine-Pt complexes enter the cell more readily than CDDP itself, but are less toxic [9]. In vivo, the thiol tripeptide glutathione (GSH) has also been found to protect the kidney against CDDP, without reducing its therapeutic efficacy [10], but the biochemical mechanism of this effect has not been elucidated.

A central role in GSH-dependent protection against the nephrotoxicity of CDDP can be envisaged for gamma-glutamyl transpeptidase (GGT), a cell membrane enzyme that is highly expressed in the microvillous surface of proximal convoluted tubule cells [11]. GGT catalyses the hydrolysis of the abundant luminal GSH to glutamate and cysteinyl-glycine. We showed recently that the product of the hydrolysis of extracellular GSH by cell membrane GGT, cysteinyl-glycine, reacts quickly against CDDP, and proposed that this mechanism could be responsible for the increased resistance of cells expressing GGT to the effects of CDDP [12].

\section{Materials and methods}

\subsection{Cell line and culture conditions}

Human immortalised epithelial HK-2 cells, derived from normal proximal convoluted tubule [13], were routinely grown in a humidified $5 \% \mathrm{CO}_{2}$ atmosphere, in a medium consisting of a 1:1 (v/v) mixture of Ham's F12 and Dulbecco's modified Eagle's medium, containing $15 \mathrm{mM}$ Hepes, $21.5 \mathrm{mM} \mathrm{HCO}_{3}, 1 \mathrm{mM}$ sodium pyruvate, and supplemented with $1 \%(\mathrm{v} / \mathrm{v})$ of a mixture of $\mathrm{L}$ glutamine, penicillin, and streptomycin $(200 \mathrm{mM}, 10000$ $\mathrm{U} / \mathrm{ml}$ and $10 \mathrm{mg} / \mathrm{ml}$, respectively), $5 \mu \mathrm{g} / \mathrm{ml}$ insulin, $5 \mu \mathrm{g} /$ $\mathrm{ml}$ transferrin, $5 \mathrm{ng} / \mathrm{ml}$ sodium selenite, $5 \mathrm{pg} / \mathrm{ml} \mathrm{3,5,3^{ \prime } -}$ triiodothyronine (T3), $5 \mathrm{mg} / \mathrm{ml}$ hydrocortisone, $5 \mathrm{pg} / \mathrm{ml}$ prostaglandin $\mathrm{E}_{1}, 5 \%(\mathrm{v} / \mathrm{v})$ fetal calf serum [14].

\subsection{Determination of GGT activity}

Cell pellets from $80 \%$ confluent cultures were washed three times in Dulbecco's phosphate-buffered solutions (PBS), and suspended in ice-cold lysis buffer $(10 \mathrm{mM}$ Tris- $\mathrm{HCl}, 10 \mathrm{mM} \mathrm{NaCl}, 1 \mathrm{mM} \mathrm{MgCl} 2, \mathrm{pH}$ 7.5). After 15 min, cells were disrupted by 20 strokes of a tight-fitting glass-glass Dounce homogeniser and centrifuged at $400 \mathrm{~g}$ for $10 \mathrm{~min}$ to remove the nuclei. The supernatant was then centrifuged at $100000 \mathrm{~g}\left(60 \mathrm{~min}, 4^{\circ} \mathrm{C}\right)$ and the resulting pellet containing cell membranes was resuspended in hypotonic buffer. GGT activity was assayed using $\gamma$-glutamyl-p-nitroanilide as a substrate and glycyl-glycine (GlyGly) as a transpeptidation acceptor [15].

\subsection{Cytochemical analysis of $G G T$}

$80 \%$ confluent cell monolayers were air-dryed overnight at room temperature. After fixing (15 s) in phosphate-buffered acetone-formalin, dishes were washed twice in ice-cold double-distilled water to remove the fixative. GGT activity was revealed by using $\gamma$-glutamyl4-methoxy-2-naphtylamide as a substrate and Fast Garnet GBC as a chromogen [16].

\subsection{Determination of intracellular $G S H$}

Glutathione was assayed by an enzymatic recycling procedure adapted to the microplate reader [17]. Briefly, samples were deproteinised in cold sulphosalicylic acid $(1 \%(\mathrm{w} / \mathrm{v})$ final concentration) and then diluted $1: 10$ in distilled water. The microtitre plate was prepared by pipetting $50 \mu \mathrm{l}$ aliquots of samples, GSH standards or blanks per well. Reagent buffer $(100 \mu 1)$ was then added containing 5,5'-dithiobis(2-nitrobenzoic acid) (0.15 $\mathrm{mM})$, NADPH $(0.2 \mathrm{mM})$ and GSH reductase $(1.0 \mathrm{U} /$ $\mathrm{ml}$ ). Plates were then immediately placed into the microplate reader (Labsystem Multiskan Plus MKII, Labsystem, Milan, Italy), and the increase of 
absorbance at $405 \mathrm{~nm}$ was monitored for $3 \mathrm{~min}$. Results were expressed as $\mathrm{nmol} \mathrm{GSH} / \mathrm{mg}$ protein of the original sample.

\subsection{Effects of co-administration of GSH on CDDP toxicity}

To exclude the possible interference of the sulphurcontaining amino acids cystine and methionine present in standard culture media, incubations were performed in sulphur amino acids-free (SAF) Roswell Park Memorial Institute (RPMI) 1640 medium (Sigma, Milan, Italy). Cells plated $24 \mathrm{~h}$ in advance into 96-well microtitre plates were incubated for $1 \mathrm{~h}$ in SAF medium containing $150 \mu \mathrm{mol} / \mathrm{l} \mathrm{CDDP}$ alone or in the presence of GSH ( 1 or $5 \mathrm{mM})$. Cisplatin cytotoxicity was judged by its antiproliferative action. Incubations with GSH were performed in the presence and in the absence of the dipeptide GlyGly $(20 \mathrm{mM})$, added as an acceptor for the transpeptidation reaction [15] to replace the physiological acceptor cystine [18]. At the end of a 1-h incubation, the growth medium was replaced with fresh medium and growth inhibition was assayed $48 \mathrm{~h}$ later by means of the WST-1 assay (Roche, Mannheim, Germany), which is based on the reduction to formazan of the tetrazolium salt 4-[3-(4Iodophenyl)2-(4-nitrophenyl)-2H-5-tetrazolio]-1,3-benzene disulphonate by viable cells. The concentration of formazan was measured at $452 \mathrm{~nm} 2 \mathrm{~h}$ after the addition of the WST-1 reagent using a microplate reader (Labsystem Multiskan Plus MKII, Labsystem, Milan, Italy).

\subsection{CDDP-dependent thiol oxidation}

The reaction of CDDP with GSH is known to form bidentate adducts, in which CDDP becomes bound to the sulphur atoms and the amide nitrogens of the cysteine residues of two GSH molecules [19]. The reactivity of thiols against equimolar concentrations $(2: 1)$ of CDDP was assayed in Dulbecco's PBS containing 1.5 $\mathrm{mM}$ CDDP and $3 \mathrm{mM}$ of cysteinyl-glycine or GSH. The latter was also incubated with CDDP in the presence of $200 \mathrm{mU} / \mathrm{ml}$ of purified bovine kidney GGT (Sigma, USA) in the presence or in the absence of the noncompetitive GGT inhibitor, acivicin (1 $\mathrm{mM})$. Incubations with GGT were performed in the presence of the dipeptide GlyGly $(20 \mathrm{mM})$, thus replacing the optimal requirements for transpeptidation [15]. Preliminary experiments had shown that the velocity of thiol oxidation by CDDP in solutions containing GSH (in the absence of GGT) or cysteinyl-glycine is not affected by GlyGly. In all cases, the disappearance of $-\mathrm{SH}$ groups was monitored at time intervals by use of the Ellman's reagent [20], and expressed as percent $-\mathrm{SH}$ equivalents of the initial content.
2.7. CDDP precomplexing with thiols: analysis of the influence of GGT on the CDDP antiproliferative effect, CDDP cellular accumulation and DNA platination

CDDP (1.5 mM in PBS), was incubated in tightly closed tubes at $37^{\circ} \mathrm{C}$, alone or with one of the following (3 mM): GSH; GSH plus GGT $(200 \mathrm{mU} / \mathrm{ml})$; cysteinylglycine. GlyGly, the acceptor for GGT-catalysed transpeptidation, was added to PBS before other components in all of the incubations ( $20 \mathrm{mM})$. After 2 and $48 \mathrm{~h}$ of incubation, samples were withdrawn for determination of the CDDP residual antiproliferative effect. Briefly, aliquots were diluted in SAF medium to obtain a final concentration of $150 \mathrm{mM}$ CDDP, and HK-2 cells, previously seeded in 96-well microtitre plates, were treated for $1 \mathrm{~h}$. The medium was then withdrawn and replaced with fresh growth medium, and after $48 \mathrm{~h}$ the WST-1 assay was performed as described above.

For determination of platinum uptake and DNA platination, after $2 \mathrm{~h}$ of precomplexing, the CDDP-thiol mixtures were diluted in SAF medium to obtain a final concentration of $150 \mu \mathrm{M}$ CDDP, and added to $100 \mathrm{~cm}^{2}$ dishes containing $80 \%$ confluent monolayers. After a 1-h incubation, dishes were quickly washed in ice-cold Dulbecco's PBS and air-dried. Analysis of platinum accumulation was then performed using atomic absorption spectrometry as previously described in Ref. [21] For determination of DNA platination, after treatment cells were detached by trypsin-ethylene diamine tetraacetic acid (EDTA) and washed in cold Dulbecco's PBS. Cell pellets were then resuspended in $500 \mu \mathrm{l}$ Tris$\mathrm{HCl}(10 \mathrm{mM}, \mathrm{pH} 8.0)$ containing $\mathrm{NaCl}(100 \mathrm{mM})$ and EDTA $(1 \mathrm{mM})$. After addition of $20 \%(\mathrm{v} / \mathrm{v})$ Triton X-100 $(25 \mu \mathrm{l})$ and proteinase $\mathrm{K}(100 \mu \mathrm{g})$ (Promega), samples were incubated overnight at $37^{\circ} \mathrm{C}$. Cells were then incubated $\left(1 \mathrm{~h}, 37^{\circ} \mathrm{C}\right)$ with RNAse $(50 \mu \mathrm{g} / \mathrm{ml})$, and DNA was purified with a phenol/chloroform extraction according to standard methods. DNA platination was assessed by inductively coupled mass spectrometry as previously described Ref. [21].

\subsection{Statistical analysis}

Statistical analysis of the data was performed by ANOVA; the statistical significance of the difference between groups was assessed by the method of Bonferroni.

\section{Results}

\subsection{GGT expression and activity in HK-2 cells}

HK-2 cells showed a very high activity of membranebound GGT (412.6 mU/mg protein). Cytochemical staining for GGT activity allowed visualisation of the enzyme activity in the plasma membrane (Fig. 1). 


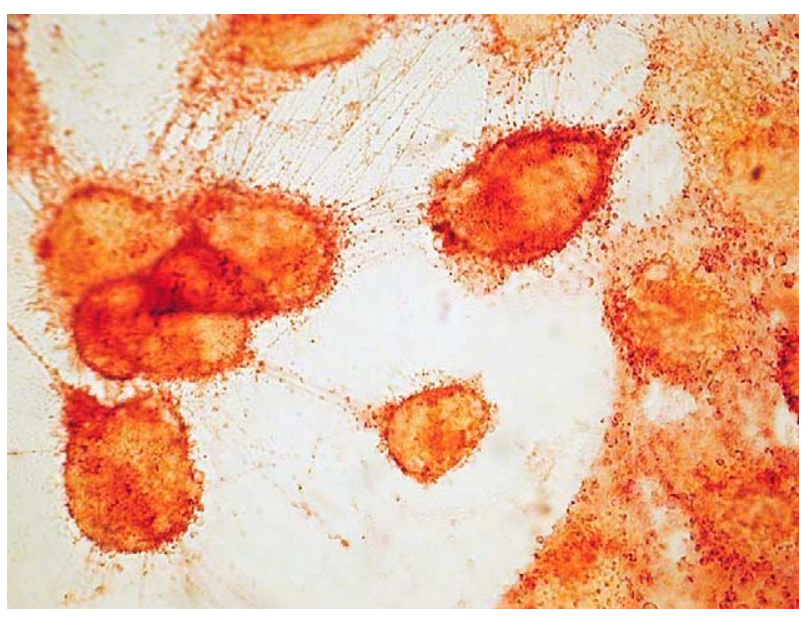

Fig. 1. Cytochemical staining for GGT activity of HK-2 cell monolayers: the darker stained deposits mark the localisation of GGT activity in the cell membranes.

\subsection{Influence of extracellular GSH on the antiproliferative effect of $C D D P$}

The influence of extracellular GSH on the antiproliferative effect of CDDP was assessed in the absence or in the presence of GSH (1 or $5 \mathrm{mM}$ ).

When cells were exposed to CDDP alone $(150 \mu \mathrm{M})$, the growth inhibition reached approximately $88 \%$ (Fig. 2). The co-incubation with GSH (1 mM) did not influence CDDP-induced growth inhibition. However, when GlyGly was also added, thereby replacing the

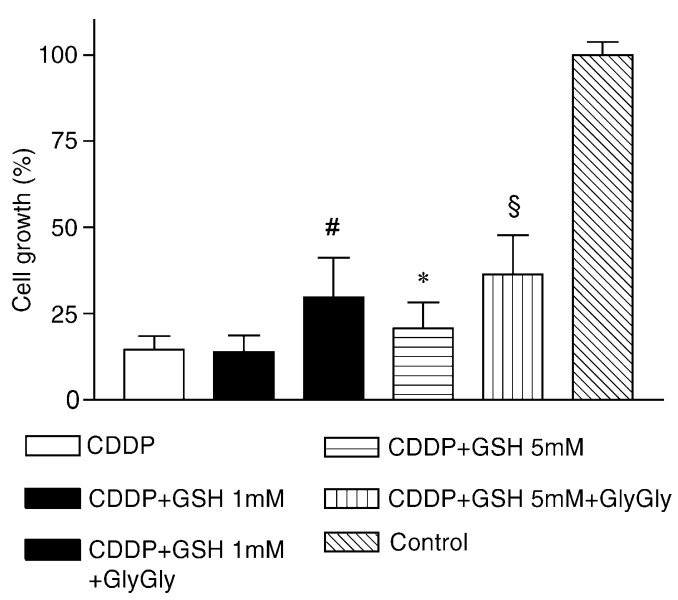

Fig. 2. Influence of GSH catabolism on cell growth inhibition by CDDP. Cell monolayers were challenged for $1 \mathrm{~h}$ with CDDP $150 \mu \mathrm{M}$, either alone or in the presence of GSH (1 or $5 \mathrm{mM})$, with or without the transpeptidation acceptor GlyGly. Cell growth was assessed by the WST-1 assay and expressed as \% cell growth with respect to the controls treated with vehicle alone. Bars are means \pm standard deviation (S.D.) $\quad(n=6) . \quad \# P<0.001$ versus CDDP alone and versus $\mathrm{CDDP}+\mathrm{GSH} 1 \mathrm{mM}, P<0.05$ versus $\mathrm{CDDP}+\mathrm{GSH} 5 \mathrm{mM} . * P<0.05$ versus $\mathrm{CDDP}$ and versus $\mathrm{CDDP}+\mathrm{GSH} 1 \mathrm{mM} . \S P<0.001$ versus CDDP, CDDP + GSH $1 \mathrm{mM}$, and versus CDDP + GSH $5 \mathrm{mM}$. All treatments were significantly different $(P<0.001)$ with respect to controls treated with vehicle alone. optimal requirements for transpeptidation, the CDDP effect was counteracted significantly, with a cell growth more than 2-fold higher than with the CDDP alone. With higher GSH concentrations $(5 \mathrm{mM})$, a direct protective effect was observed, but again the addition of GlyGly still increased cell growth $(20.7 \pm 7.4 \%$ versus $36.3 \pm 11.1 \%$; Fig. 2). In preliminary experiments (data not shown), GlyGly did not influence cell growth per se and did not modulate the CDDP growth inhibitory effect, thus confirming that enhancement of the protective effect of GSH in the presence of GlyGly was exclusively due to increased GGT-mediated GSH hydrolysis.

At the end of CDDP treatment, the intracellular concentration of GSH was found to be slightly higher in monolayers co-incubated with CDDP and GSH compared with controls and cells exposed to CDDP alone. When GlyGly was also added, no further increase, but rather a decrease, of intracellular GSH was found, thus indicating that the protection against CDDP offered by the enhancement of GGT activity with GlyGly did not depend on increased intracellular GSH synthesis (Table 1).

\subsection{Reactivity of thiols with CDDP}

To provide additional support for a role of GSH metabolism in the protective action, we investigated the reactivity of the thiol residue of cysteinyl-glycine against CDDP compared with that of GSH. The reaction of CDDP with thiols was monitored by following the oxidation of $-\mathrm{SH}$ groups in incubations containing $1.5 \mathrm{mM}$ CDDP and an equimolar concentration $(3 \mathrm{mM})$ of the thiol tested. To avoid any interference by other amino acids, incubations were performed in Dulbecco's PBS, which reproduces the conditions of high $\mathrm{Cl}^{-}$concentrations of RPMI 1640 culture medium and of the extracellular environment in vivo. The initial velocity of $-\mathrm{SH}$ disappearance in the presence of CDDP was relatively low for GSH, and approximately 10 times higher for the

Table 1

Intracellular GSH content of HK-2 cells at the end of the exposure to CDDP in cytotoxicity assays

\begin{tabular}{lc}
\hline & $\begin{array}{c}\text { Intracellular GSH } \\
\text { (nmol/mg protein) }\end{array}$ \\
\hline Control & $29.4 \pm 5.2 \$$ \\
CDDP & $25.21 \pm 1.4^{*} \#$ \\
CDDP + GSH 1 mM & $37.3 \pm 2.03^{*} \$$ \\
CDDP + GSH 1 mM + GlyGly & $34.25 \pm 1.2 \#$ \\
\hline
\end{tabular}

Intracellular GSH was assessed after incubating cell monolayers for 1 $\mathrm{h}$ in SAF medium containing $150 \mu \mathrm{M}$ CDDP alone or in combination with GSH $1 \mathrm{mM}$, with or without GlyGly $20 \mathrm{mM}$. Data are the mean \pm standard deviation (S.D.) of three separate determinations. Treatments marked with the same symbol were significantly different $(P<0.05)$. GSH, glutathione; CDDP, cis-diammine-dichloro-platinum; GlyGly, glycyl-glycine; SAF, sulphur amino acids-free. 


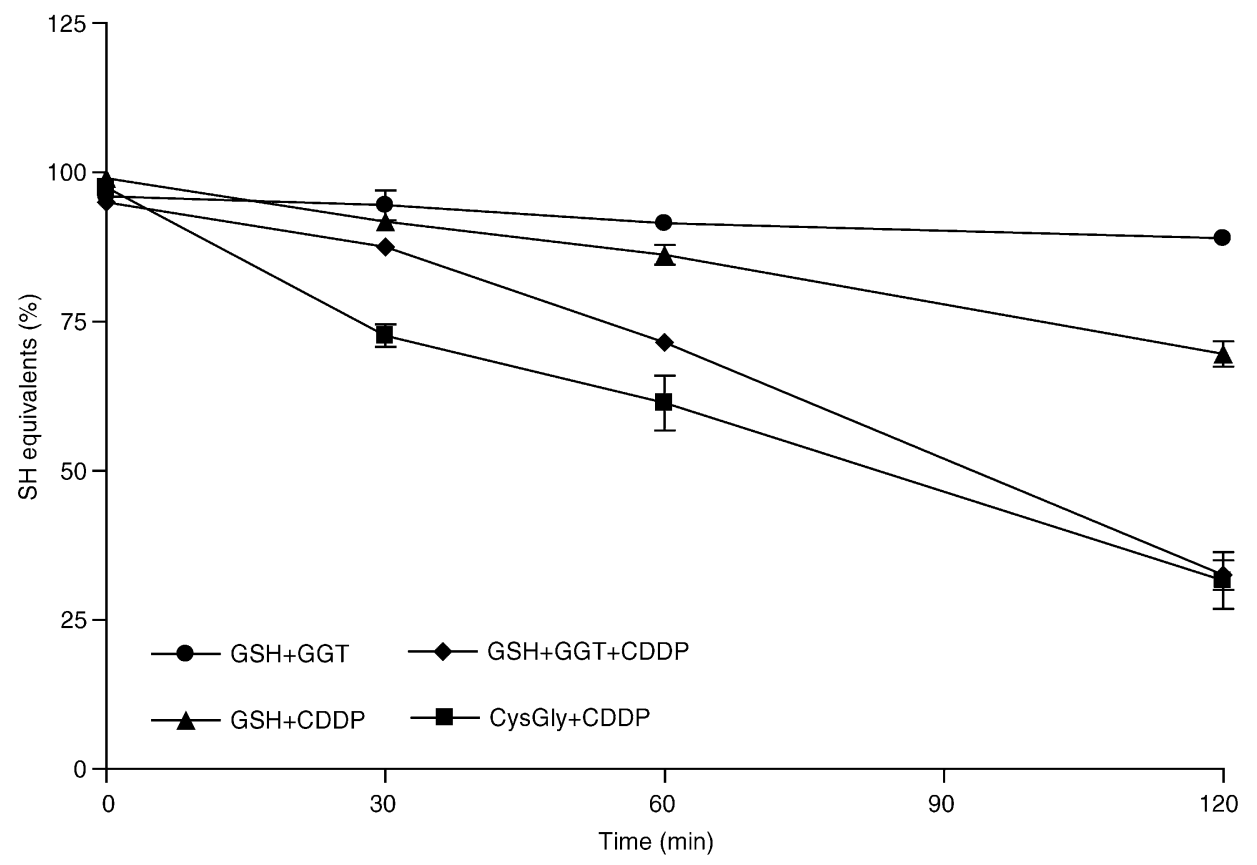

Fig. 3. Reactivity of thiols with CDDP. The content of $-\mathrm{SH}$ groups was assessed at time intervals in a mixture containing $1.5 \mathrm{mM}$ CDDP and 3 $\mathrm{mM}$ cysteinyl-glycine or GSH. Each experimental point is the mean \pm S.D. of three separate determinations.

GGT-derived GSH catabolite cysteinyl-glycine. When GGT and GlyGly were added to GSH, the disappearance of $-\mathrm{SH}$ groups proceeded substantially at the same rate as in the presence of preformed cysteinylglycine (Fig. 3). In these conditions, the addition of the GGT inhibitor acivicin to a mixture containing GSH prevented - $\mathrm{SH}$ oxidation, thus confirming that the enhanced $-\mathrm{SH}$ loss in the presence of GGT was due selectively to enzyme activity and not to contaminants in the enzyme preparation (not shown).

\subsection{Influence of precomplexing with thiols on the CDDP antiproliferative effect, cellular accumulation and DNA platination}

To assess whether the extracellular complexing of CDDP with thiols could be responsible for the inactivation of CDDP by GGT, the effects of precomplexing of CDDP in the presence of equimolar concentrations of GSH, cysteinyl-glycine, or GSH plus GGT on the antiproliferative effects of the drug, drug accumulation as well as on DNA platination was monitored. Although a modest decrease of antiproliferative activity was observed when CDDP alone was incubated at $37^{\circ} \mathrm{C}$ in PBS, likely due to the spontaneous decay of the drug, after $48 \mathrm{~h}$ pre-incubation with GSH or cysteinyl-glycine, when all thiols had completely reacted with CDDP (data not shown), the drug antiproliferative effect was completely abolished. This was independent of the thiol used for the precomplexing (Fig. 4a). After $2 \mathrm{~h}$ preincubation with cysteinyl-glycine, when approximately $70 \%$ of the thiols had reacted with CDDP (Fig. 3), the growth inhibition due to CDDP treatment was lost, while the same time of precomplexing with GSH alone, which corresponded to $30 \%$ thiol oxidation, resulted in only a partial loss of the antiproliferative effect of CDDP. When CDDP was precomplexed with GSH in the presence of GGT and GlyGly, the loss of antiproliferative action was comparable to precomplexing with CysGly (Fig. 4b). Platinum uptake and DNA platination in cells exposed to 2 -h precomplexed mixtures of CDDP and thiols followed the same trends, the most effective inhibition of platinum uptake and platinumDNA adduct formation being observed by precomplexing of CDDP with cysteinyl-glycine or a mixture of GSH, GGT and GlyGly. Platinum uptake and DNA platination were decreased to a lesser extent when CDDP was precomplexed with GSH alone (Table 2).

\section{Discussion}

We investigated the mechanism of the protective action of exogenous GSH against the CDDP-induced nephrotoxicity by using the immortalised cell line, HK2. The precise concentration that CDDP attains within the proximal convoluted tubule after administration is not known, and considering that the glomerular filtrate is concentrated several-fold within this portion of the nephron, and CDDP is known to be concentrated within the kidney [4-6], a CDDP concentration higher than therapeutic plasma levels of CDDP [22] was chosen for all of the experiments. In contrast to previous 


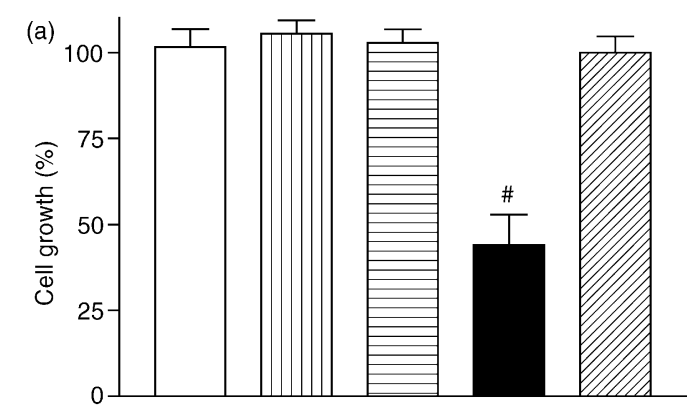

Treatment

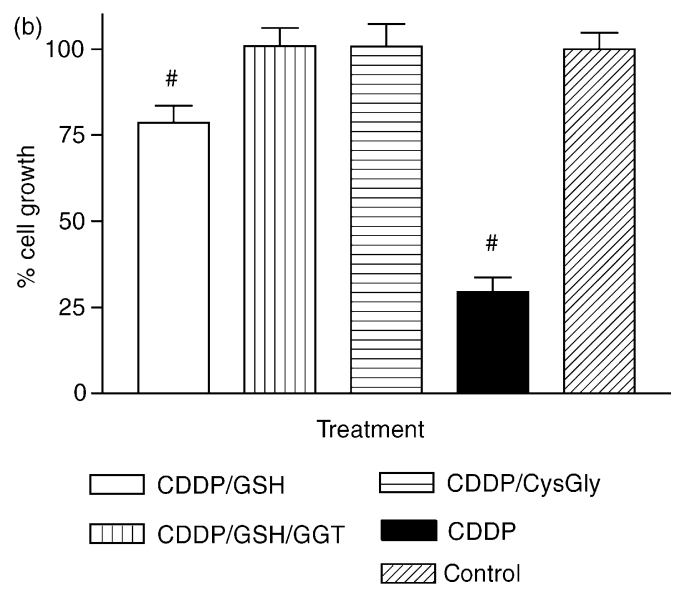

Fig. 4. Effect of precomplexing with biological thiols on the antiproliferative activity of CDDP: $1.5 \mathrm{mM}$ CDDP was pre-incubated with $3 \mathrm{mM}$ cysteinyl-glycine or GSH, the latter with or without 100 $\mathrm{mU} / \mathrm{ml}$ bovine kidney GGT. The transpeptidation acceptor glycl-glycine (GlyGly) $(20 \mathrm{mM})$ was present in all mixtures. After precomplexing, the mixture was diluted in SAF medium to reach a final concentration of $150 \mathrm{uM}$ platinum, and cell proliferation assayed $48 \mathrm{~h}$ later by WST-1. In (a), the proliferation assay was performed after 48 $\mathrm{h}$ precomplexing. In (b), after $2 \mathrm{~h}$ precomplexing. Cell growth was assessed by the WST-1 assay and expressed as \% cell growth with respect to controls treated with vehicle alone. Bars are means \pm S.D. $(n=10)$. $\# P<0.001$ versus all other treatments and versus controls treated with PBS alone.

experiments in HeLa cells conducted with lower platinum concentrations [12], the supplementation of incubation medium with GSH - despite the higher GGT activity of HK-2 cells with respect to $\mathrm{HeLa}$ - had a relatively modest protective action, a statistically significant reduction of the antiproliferative effect of CDDP being observed only in the presence of high GSH concentrations $(5 \mathrm{mM})$. However, antiproliferative tests were performed in SAF medium, which lacks cystine, the best physiological acceptor for the GGT-catalysed transpeptidation of GSH, in the absence of which the breakdown of GSH is severely inhibited [18]; when the non-thiol-containing transpeptidation acceptor GlyGly was introduced into the medium used for antiproliferative assays, a significant protection of HK-2 cells against CDDP toxicity was observed, even with GSH $1 \mathrm{mM}$. This finding confirms the hypothesis that
Table 2

influence of GGT activity on platinum uptake and on the formation of platinum-DNA adducts

\begin{tabular}{lcc}
\hline Treatment & $\begin{array}{l}\text { Platinum uptake } \\
\text { (ng/106 cells) }\end{array}$ & $\begin{array}{l}\text { DNA platination } \\
\text { (pg Pt/ug DNA) }\end{array}$ \\
\hline CDDP & $19.2 \pm 0.7^{*}$ & 25.21 \\
CDDP + GSH & $11.96 \pm 0.6^{*}$ & 14.82 \\
CDDP + CysGly & $7.09 \pm 0.8 \#$ & 6.32 \\
CDDP + GSH + GGT & $8.69 \pm 0.9 \#$ & 10.97 \\
Control & N.D. & N.D.
\end{tabular}

CDDP was pre-complexed for $2 \mathrm{~h}$ at $37{ }^{\circ} \mathrm{C}$ with $3 \mathrm{mM}$ pre-formed CysGly or with GSH in the presence or in the absence of $100 \mathrm{mU} / \mathrm{ml}$ bovine kidney GGT. $80 \%$ confluent cell monolayers were exposed to the mixture, diluted in SAF culture medium to reach a final concentration of $150 \mu \mathrm{M}$ platinum. After $1 \mathrm{~h}$, intracellular platinum and DNA-platinum adducts were assessed. Platinum uptake data are the mean \pm S.D. $(n=3) . * P<0.01$ versus all other treatments and between them; $\# P<0.01$ versus all other treatments, but not between them. A representative experiment is reported, repeated experiments gave comparable results. As for DNA platination, the data derive from a single experiment, which was repeated with comparable results. GGT, $\gamma$-glutamyl transpeptidase; CDDP, cis-diammine-dichloro-platinum; GSH, glutathione; CysGly, cysteinyl-glycine; ND, not detectable.

the protective effect of GSH against CDDP is mediated by its extracellular catabolism by GGT.

GGT provides cells with a secondary source of cysteine derived from the breakdown of extracellular GSH [11], and this can thus favour the reconstitution of intracellular GSH, that is considered to play a major role in the cellular defence against nucleophilic agents like CDDP and other alkylating agents [23]. However, it is unlikely that in HK-2 cells the observed protection offered by GSH and efficient GGT activity is due to an enhancement of intracellular GSH synthesis.

In fact, despite decreasing CDDP efficacy, supplementation of SAF medium with GlyGly did not result in a further increase of intracellular GSH contents above the levels seen in cells exposed to CDDP and GSH alone. Furthermore, kidney tubule cells are capable of transporting intact GSH, thus limiting the influence of GGT on intracellular GSH levels [24]. This feature of renal tubule cells would also explain the significant increase of intracellular GSH observed upon GSH administration in co-incubation experiments, which was not enhanced by GlyGly supplementation (Table 1).

GSH reacts with CDDP in a 2:1 ratio to form a complex that is a symmetrical bidentate adduct of 809 $\mathrm{D}$, with both the sulphur and the amide nitrogen of the cysteine residues bound to CDDP, trans to each other [19]. Such reaction is, however, remarkably slow $[12,24,25]$, while we have recently shown that the formation of the adduct between CDDP and cysteinyl-glycine is approximately 10 times quicker [12]. The present results provide direct evidence that both the CDDPGSH and CDDP-cysteinylglycine complexes lack an antiproliferative activity, but the more rapid reactivity 
of cysteinylglycine with CDDP allows a more rapid loss of drug activity, as assessed by antiproliferative activity tests; GGT enzyme activity appears to trigger the inactivation of CDDP in the presence of extracellular GSH by converting the otherwise poorly reactive GSH into the highly reactive CysGly; these data confirm the original hypothesis that proposed a key role for GGT in the protective effect against the nephrotoxicity of CDDP observed following GSH administration.

It has been shown recently that extracellular sulphur amino acids, in particular cysteine, can reduce CDDP cytotoxicity in cultured renal tubule cells due to the formation of a CDDP/cysteine complex which was found to be more easily absorbed, but less toxic than CDDP itself [9]. These observations are in line with our present results, showing that $\mathrm{CDDP} / \mathrm{GSH}$ and $\mathrm{CDDP} /$ cysteinyl-glycine are less toxic than the parental drug. However, in our experiments, precomplexing of CDDP with both GSH and cysteinyl-glycine resulted in a decreased intracellular accumulation and decreased DNA platination suggesting a defective transport of thiol-CDDP complexes and a reduced reactivity of these complexes with the primary intracellular target. Further studies are needed to understand whether the reduced absorption of the thiol-CDDP complex is a peculiar feature of HK-2 cells or a peculiar property of the GSH and cysteinylglycine conjugates of CDDP. The fact that the same complex has been found in the supernatant of CDDP-resistant HeLa cells transfected with the full-length cDNA coding for human GGT [12] seems to support the latter hypothesis.

Conflicting results have been previously reported regarding the role of renal tubular GGT in CDDP nephrotoxicity in vivo. In fact, transgenic mice lacking the GGT gene, as well as mice treated with the GGT inhibitor, acivicin, have a reduced mortality rate and show less nephrotoxicity after treatment with a dose of CDDP that causes the death of approximately half of control wild-type animals [26]. These results led Hanigan and coworkers to propose the hypothesis that the sequential extracellular hydrolysis catalysed by GGT and membrane dipeptidase of the GSH-platinum complex formed intracellularly leads to the formation of a cysteine-platinum complex; the hydrolysis of this complex by intracellular cysteine-conjugate $\beta$-lyase (CCBL) would cause the formation of an active thiol, the ultimate compound responsible for the renal toxicity of CDDP. In line with this hypothesis, Towsend and Hanigan have shown that in mice the administration of the CCBL inhibitor aminooxyacetic acid reduces the toxicity of a sublethal dose of CDDP [27]. Anyway, Rooseboom and coworkers have recently found that transfection of LLC-PK1 cells, which have intrinsically low CCBL activity, with the full-length cDNA coding for CCBL did not increase, but on the contrary decreased the antiproliferative effect of CDDP [28], thus providing strong evidence against the above hypothesis that GGT enhances CDDP nephrotoxicity.

We propose an alternative explanation for the protective effect of GGT inhibition against CDDP nephrotoxicity in vivo: it is well known that after acivicin administration [29], as well as in GGT knockout mice [30], GSH concentrations are known to increase severalfold in plasma; this fact, which increases glomerular filtration of GSH, in association with the reabsorption of water and the active transport of GSH into the proximal convoluted tubule lumen increases the urinary concentration of GSH to $5-30 \mathrm{mmol} / 1$ [11], a concentration that we found provides direct protection against CDDP cytotoxicity.

In conclusion, extracellular breakdown of GSH by GGT, and the consequent release of the highly reactive dipeptide thiol cysteinyl-glycine, is likely to be a major determinant of the protective action exhibited by exogenous GSH against CDDP damage to renal cells. The relevance of this observation may extend beyond CDDP nephrotoxicity. Most human tumours with inherent resistance to CDDP present with elevated GGT activity [31], and GSH/GGT-dependent inactivation of CDDP might also be involved in drug resistance in tumours [12]. A better knowledge of the physiological and pathological determinants of this kind of interaction between CDDP and extracellular thiols could lead to significant improvements in the outcome of CDDP therapy, in terms of both a reduction of unwanted toxicity and an increase in drug efficiency.

\section{Acknowledgements}

The financial support of A.I.R.C. (Associazione Italiana Ricerca sul Cancro, Milan, Italy) is gratefully acknowledged. Additional funds were derived from the Italian Ministry for Education and Research 'FIRB 2002 Funds'. M. Sotiropoulou was the recipient of a bursary from a joint programme between Italian and Greek Universities.

\section{References}

1. Rosenberg B. Fundamental studies with cisplatin. Cancer 1985, 55, 2303-2316.

2. Pinto AL, Lippard SJ. Binding of the antitumor drug cis-diamminedichloroplatinum(II) (cisplatin) to DNA. Biochim Biophys Acta 1985, 780, 167-180.

3. Chu G. Cellular responses to cisplatin. J Biol Chem 1994, 269, 787-790.

4. Daugaard G, Abildgaard U. Cisplatin nephrotoxicity. A review. Cancer Chemother Pharmacol 1989, 25, 1-9.

5. Blachley JD, Hill JB. Renal and electrolyte disturbances associated with cisplatin. Ann Int Med 1981, 95, 628-632. 
6. Bogin E, Marom M, Levi Y. Changes in serum, liver, and kidneys of cisplatin treated rats; effect of antioxidants. Eur J Clin Chem Clin Biochem 1994, 32, 843-851.

7. Deegan PM, Pratt IS, Ryan MP. The nephrotoxicity, cytotoxicity, and renal handling of a cisplatin methionine complex in male wistar rats. Toxicology 1994, 89, 1-14.

8. Appenroth D, Winnefeld K, Schhroter H, Rost M. Beneficial effect of acetylcysteine on cisplatin nephrotoxicity in rats. $J$ Appl Toxicol 1993, 13, 189-192.

9. Kröning R, Lichtenstein AK, Nagami GT. Sulfur-containing amino acids decrease cisplatin cytotoxicity and uptake in renal tubule epithelial cell lines. Cancer Chemother Pharmacol 2000, 45, 43-49.

10. Zunino F, Pratesi G, Micheloni A, et al. Protective effect of reduced glutathione against cisplatin-induced renal and systemic toxicity and its influence on the therapeutic activity of the antitumor drug. Chem Biol Interac 1989, 70, 89-101.

11. Meister A. Metabolism and transport of glutathione and other $\gamma$-glutamyl compounds. In Larsson A, Orrenius S, Holmgren A, Mannervik B, eds. Functions of Glutathione: Biochemical, Toxicological and Clinical Aspects. New York, Raven Press, 1983, 122.

12. Daubeuf S, Leroy P, Paolicchi A, et al. Enhanced resistance of HeLa cells to cisplatin by overexpression of $\gamma$-glutamyltransferase. Biochem Pharmacol 2002, 64, 207-216.

13. Ryan MJ, Johnson G, Fuerstemberg SM, Zager RA, TorokStorb B. HK-2, an immortalized proximal tubule epitelial cell line from normal adult human kidney. Kidney Int 1994, 45, 48-57.

14. Tramonti G, Romiti N, Norpoth M, Chieli E. P-Glycoprotein in HK-2 proximal tubule cell line. Ren Fail 2001, 23, 331-337.

15. Huseby NE, Stromme JH. Practical points regarding routine determination determination of $\gamma$-glutamyltransferase $(\gamma-\mathrm{GT})$ in serum with a kinetic method at $37^{\circ} \mathrm{C}$. Scand J Clin Lab Invest 1979, 34, 357-363.

16. Khalaf MR, Hayhoe FGJ. Cytochemistry of $\gamma$-glutamyltransferase in haemic cells and malignancies. Histochem $J$ 1987, 19, 385395.

17. Baker MA, Cerniglia GJ, Zaman A. Microtiter plate assay for the measurement of glutathione and glutathione disulfide in large numbers of biological samples. Anal Biochem 1990, 190, 360-365.

18. Allison DR, Meister A. Evidence that transpeptidation is a significant function of $\gamma$-glutamyltranspeptidase. J Biol Chem 1981, 256, 2988-2992.

19. Dedon PC, Borch RF. Characterization of the reactions of plati- num antitumor agents with biologic and nonbiologic sulfur-containing nucleophyles. Biochem Pharmacol 1987, 36, 1955-1964.

20. Deakin H, Ord MG, Stocken LA. Glucose 6-Phosphate-dehydrogenase activity and thiol content of thymus nuclei from control and X-irradiated rats. Biochem J 1963, 89, 296-301.

21. Perego P, Caserini C, Gatti L, et al. A novel trinuclear platinum complex overcomes cisplatin resistance in an osteosarcoma cell system. Mol Pharmacol 1999, 55, 528-534.

22. Erdlenbruch B, Nier M, Kern W, Hiddemann W, Pekrun A, Lacomekk N. Pharmacokinetics of cisplatin and relation to nephrotoxicity in paediatric patients. Eur J Clin Pharmacol 2001, 57, 393-402.

23. Godwin AK, Meister A, O'Dwyer PJ, Huang CS, Hamilton TC, Anderson ME. High resistance to cyplatin in human ovarian cancer cell lines is associated with marked increase of glutathione synthesis. Proc Natl Acad Sci USA 1992, 89, 3070-3074.

24. Hagen TM, Aw TY, Jones DP. Glutathione uptake and protection against oxidative injury in isolated kidney cells. Kidney Int 1988, 34, 74-81.

25. Ishikawa T, Ali-Osman F. Glutathione-associated cis-diamminedichloroplatinum (II) metabolism and ATP-dependent efflux from leukemia cells. $J$ Biol Chem 1993, 268, 20116-20125.

26. Hanigan MH, Lykissa ED, Townsend DM, Ou CN, Barrios R, Lieberman MW. Gamma-glutamyl transpeptidase-deficient mice are resistant to the nephrotoxic effects of cisplatin. Am J Pathol 2001, 159, 1889-1894

27. Towsend DM, Hanigan MH. Inhibition of $\gamma$-glutamyl transpeptidase or cysteine S-conjugate- $\beta$-lyase activity blocks the nephrotoxicity of cysplatin in mice. $J$ Pharmacol Exp Ther 2002, 300, 142-148.

28. Rooseboom M, Schaaf G, Commandeur JNM, Vermeulen NPE, Fink-Gremmels J. $\beta$-Lyase-dependent attenuation of cysplatinmediated toxicity by selenocysteine $S e$-conjugates in renal tubular cell lines. J Pharmacol Exp Ther 2002, 301, 884-892.

29. Griffith OW, Meister A. Translocation of intracellular glutathione to membrane-bound $\gamma$-glutamyltranspeptidase as a discrete step in he $\gamma$-glutamyl cycle: glutathionuria after inhibition of transpeptidase. Proc Natl Acad Sci USA 1979, 76, 268-272.

30. Liebermann MW, Wiseman AL, Shi Z-Z, et al. Growth retardation and cysteine deficiency in $\gamma$-glutamyl transpeptidase-deficient mice. Proc Natl Acad Sci USA 1996, 93, 7923-7926.

31. Pratesi G, Dal Bo L, Paolicchi A, Tonarelli P, Tongiani R, Zunino F. The role of the glutathione-dependent system in tumor sensitivity to cisplatin: a study of human tumor xenografts. Ann Oncol 1995, 6, 283-289. 